\title{
The impact of COVID-19 on clinical education of internal medicine trainees
}

\author{
Brian Gaffney ${ }^{1}$ (D) . Orla O'Carroll ${ }^{1} \cdot$ Finbarr Conroy ${ }^{1} \cdot$ Marcus W. Butler $^{1,2} \cdot$ Michael P. Keane $^{1,2} \cdot$ Cormac McCarthy $^{1,2,3}$
}

Received: 11 August 2020 / Accepted: 13 August 2020 / Published online: 15 August 2020

(C) Royal Academy of Medicine in Ireland 2020

The World Health Organization classified the COVID-19 outbreak as a pandemic on March 11, 2020. Efforts to combat the spread of this disease resulted in significant changes to daily life and in particular to the structure of healthcare systems and postgraduate medical training. In Ireland, postgraduate basic speciality training (BST) in general internal medicine consists of a 2-year programme of supervised clinical training posts. Training encompasses rotations through several subspecialties coordinated by the Royal College of Physicians of Ireland (RCPI). To complete, the programme trainees must attend subspecialty outpatient clinics, fulfil acute on-call duties, perform directly observed procedures, attend formal teaching sessions and complete specific curriculum courses.

St Vincent's University Hospital is an 836-bed tertiary referral academic health centre in Dublin, Ireland. At the outset of this pandemic, significant restructuring of hospital services was undertaken to protect acute care services. To appropriately identify COVID-19 cases in an infection control environment and improve the flow of patients, a specific COVID-19 triage pathway was established, and the traditional emergency department (ED) continued in parallel to deal with cases that had very low suspicion of COVID-19. Previously most patients would be seen initially by a member of the ED team and where appropriate were referred to the internal medicine service. As a consequence of the need to preserve physical distancing in the ED an emphasis was placed on the timely and safe movement of patients through this initial ED-housed assessment onto their assigned in-hospital beds. This resulted in a significant change in the type and burden of work, with BST

Cormac McCarthy

Cormac.McCarthy@UCD.ie

1 Department of Respiratory Medicine, St. Vincent's University Hospital, Dublin 4, Ireland

2 School of Medicine, University College Dublin, Dublin 4, Ireland

3 Education and Research Centre, St Vincent's University Hospital, University College Dublin, Dublin 4, Ireland trainees partaking in the ED triage to allow improved flow, resulting in no referral system to internal medicine.

Notably, there has been a change in the variety of presentations assessed by medical trainees through the formation of these parallel assessment pathways. A set of referral criteria were adopted, based on national and international public health guidance, aimed to designate patients to either pathway or these reflected dynamic changes in the national case definition of COVID-19. Table 1 illustrates the most common presenting complaints which prompted triage of patients through the COVID-19 referral pathway. Comparison is made between the frequency of these presentations over a weeklong period prior to the pandemic and during the surge experienced at the authors' institution. Prior to the pandemic, an average of 30 patients in a 24-h period were referred for assessment and potential admission by general internal medicine in the authors' institution. The majority (93.3\%) of these assessments resulted in hospital admission. During the COVID19 pandemic, an average of 52 patients were assessed per 24-h period, mostly with respiratory symptoms; however, much fewer assessments resulted in admission (53.8\%), amidst a prevailing culture of preserving future surge capacity (Table 1). There were statistically significant differences noted in the frequency of presentation of some of the most common symptoms referred through the COVID-19 pathway. The change in the assessment-to-admission ratio potentially reflects the above-mentioned pressures upon primary care providers during this time and the increased sensitivity required of triage processes to capture milder COVID-19 presentations during then-widespread community transmission.

During this period, the process of medical handover changed; the conventional consultant-led post-take ward round changed to a virtual 'dry' round where the emphasis was on effective dissemination of crucial information about each patient in a timely manner. This impacted significantly on the opportunities for medical trainees to learn at the bedside from senior supervising consultants and to have a forum of constructive feedback. Moreover, during this time, each ward 
Table 1 Presentations from the emergency department referred to the internal medicine team prior to the COVID-19 pandemic and during the COVID-19 pandemic

\begin{tabular}{llll}
\hline Presentation & $\begin{array}{l}\text { Pre-pandemic mean no. per day } \\
\text { (\% of total) }\end{array}$ & $\begin{array}{l}\text { Pandemic mean no. per day } \\
(\% \text { of total) }\end{array}$ & $p$ value \\
\hline Total assessments & 30 & 52 & 0.0146 \\
Number admitted & $28(93.3 \%$ of assessments) $)$ & $28(53.8 \%$ of assessments $)$ & 0.972 \\
COVID-19 positive cases & NA & 6 & NA \\
Presenting complaint (mean number of presentations per day) & & \\
Dyspnoea & $3(10 \%)$ & $16(30 \%)$ & 0.00025 \\
Cough & $2(6.7 \%)$ & $10(19 \%)$ & 0.0062 \\
Pyrexia & $2(6.7 \%)$ & $9(17.3 \%)$ & 0.0092 \\
Chest Pain & $2(6.7 \%)$ & $9(17.3 \%)$ & 0.0053 \\
Abdominal Pain & $1(3.3 \%)$ & $6(11.5 \%)$ & 0.0081 \\
Nausea, vomiting, diarrhoea & $3(10 \%)$ & $5(9.6 \%)$ & 0.79 \\
Skin rash & $1(3.3 \%)$ & $2(3.8 \%)$ & 0.82 \\
Headache & $0(0 \%)$ & $1(1.9 \%)$ & 0.71 \\
\hline
\end{tabular}

Data are mean presentations/assessments per day, and data for each column were collected over a representative 1week period in each case. Presenting complain demonstrated represents symptoms which warranted triage towards the COVID-19 pathway. $p$ values were calculated to assess for any significant differences in the presentations seen before and after COVID; Student's $t$ test was used; $p<0.05$ was significant. NA, not applicable was assigned multiple senior decision-makers to improve patient flow through the hospital, thus reducing independence of the medical trainees as key decision-makers. Perception of autonomy is an important factor in preservation of physician wellbeing and is a vital part of training for junior/trainee doctors $[1,2]$.

In tandem with the increased burden on acute care services in the authors' institution, outpatient clinics were scaled back due to redeployed staff/physical distancing/infection control constraints, and virtual telephone reviews replaced in-person patient visits. The numbers of patients attending these clinics, even in a virtual capacity, were reduced, and therefore, the opportunities for trainee engagement in the outpatient environment were significantly less. Furthermore, medical trainees were reassigned from team-based specialty rotations to nonspeciality-specific general medicine wards. This attenuated the team structure intrinsic to medical training and resulted in less exposure to specialty care, an integral part of the curriculum. Moreover, procedural training was attenuated by limitation on the number of procedures and necessary infection control measures. Formal delivery of education, which previously included specialty-specific case-based conferences, journal clubs, teaching conferences, and research meetings, has decreased as the demands on service provision have increased. However, the impact of this on trainees has been offset somewhat by the conversion of institute-wide teaching sessions to virtual lecture series accessible to all users.

Nevertheless, there have been several positive impacts on trainees because of the altered hospital environment; staffing levels were expanded to allow for the surge in demand on acute hospital care, and the camaraderie between staff facilitated the smooth transfer of tasks/handover and allowed flexibility to cross-cover shifts. Trainee doctors are experiencing increased demands at work however, through longer time spent in hospital and the increased caseload. Burnout syndrome is recognised to be associated with times of increased workload and diminished resources, and this is compounded by limited access to usual coping mechanisms employed by doctors given the physical distancing measures. This could contribute to reduced resilience in trainees [3-5].

The COVID-19 pandemic has impacted significantly on medical training, and this is likely reflected across the world in internal medicine and other speciality training programmes. Identifying and understanding the new education challenges posed by the new environment healthcare providers find themselves in will be key to maintaining a high standard of professional training for junior doctors. As a potential second wave of COVID-19 or other pandemic disease could occur in the future, it is essential to bolster medical training of the highest standard.

\section{References}

1. Raj KS (2016) Well-being in residency: a systematic review. J Grad Med Educ 8(5):674-684. https://doi.org/10.4300/JGME-D-1500764.1

2. Finn KM, Metlay JP, Chang Y et al (2018) Effect of increased inpatient attending physician supervision on medical errors, patient safety, and resident education: a randomized clinical trial. JAMA Intern 
Med 178(7):952-959. https://doi.org/10.1001/jamainternmed.2018. 1244

3. Mealer M, Moss M, Good V et al (2016) What is burnout syndrome (BOS)? Am J Respir Crit Care Med 194(1):1-2. https://doi.org/10. 1164/rccm.1941P1

4. Rodrigues H, Cobucci R, Oliveira A et al (2018) Burnout syndrome among medical residents: a systematic review and meta-analysis. PLoS One 13(11):e0206840. https://doi.org/10.1371/journal.pone. 0206840
5. Lacy BE, Chan JL (2018) Physician burnout: the hidden health care crisis. Clin Gastroenterol Hepatol 16(3):311-317. https://doi.org/10. 1016/j.cgh.2017.06.043

Publisher's note Springer Nature remains neutral with regard to jurisdictional claims in published maps and institutional affiliations. 